\title{
HERMITIAN OPERATORS ON BANACH ALGEBRAS OF LIPSCHITZ FUNCTIONS
}

\author{
FERNANDA BOTELHO, JAMES JAMISON, A. JIMÉNEZ-VARGAS, \\ AND MOISÉS VILLEGAS-VALLECILLOS \\ (Communicated by Thomas Schlumprecht)
}

\begin{abstract}
For compact metric spaces $(X, d)$, we show that the Lipschitz spaces $\operatorname{Lip}(X, d)$ and the little Lipschitz $\operatorname{spaces} \operatorname{lip}\left(X, d^{\alpha}\right)$ with $0<\alpha<1$, equipped with the sum norm, support only trivial hermitian operators, that is, real multiples of the identity operator.
\end{abstract}

\section{INTRODUCTION}

Let $A$ be a complex Banach algebra with unity $I$ and let $A^{*}$ be its dual space. Given $a \in A$, recall that the algebraic numerical range $V(a)$ is given by

$$
V(a)=\left\{F(a): F \in A^{*},\|F\|=F(I)=1\right\} .
$$

An element $a \in A$ is said to be hermitian if $V(a) \subset \mathbb{R}$. It is known that $a \in A$ is hermitian if and only if $\|\exp (i t a)\|=1$ for all $t \in \mathbb{R}$; see 3 .

Let $E$ be a complex Banach space and $\mathcal{B}(E)$ the Banach algebra of all bounded linear operators on $E$ equipped with the operator norm. It is well-known that an operator $T \in \mathcal{B}(E)$ is hermitian if and only if $\exp (i t T)$ is an isometry for each $t \in \mathbb{R}$; see [6, Theorem 5.2.6]. The set of hermitian operators on $E$ is a real subspace of $\mathcal{B}(E)$ which contains all operators of the form $\lambda I$, where $\lambda$ is a real number. A hermitian operator is said to be trivial if it is a real multiple of the identity operator.

Some important Banach spaces only support trivial hermitian operators, as for example, the Bergman spaces $L_{a}^{p}(\Delta)(1 \leq p<\infty, p \neq 2)$ [9. Corollary 5.4] and the Hardy spaces $H^{p}(\Delta)(1 \leq p<\infty, p \neq 2)$ [1. Also, the hermitian operators on several spaces of scalar-valued continuous functions defined on the interval $[0,1]$ are known to be just real scalar multiples of the identity. Such spaces include the space of continuously differentiable functions $C^{1}[0,1]$; the space of absolutely continuous functions $A C[0,1]$; and the spaces of Lipschitz functions: $\operatorname{Lip}[0,1]$ and $\operatorname{lip} \alpha, 0<\alpha<1$. We recall that $\operatorname{lip} \alpha$ consists of all period 1 functions on $\mathbb{R}$ satisfying $|f(x)-f(y)|=o\left(|x-y|^{\alpha}\right)$ uniformly as $|x-y| \rightarrow 0$; cf. [2, Theorem 3.1].

Received by the editors February 6, 2012 and, in revised form, September 5, 2012; September 13, 2012; and October 9, 2012.

2010 Mathematics Subject Classification. Primary 46E15, 47B15, 47B38.

Key words and phrases. Spaces of Lipschitz functions, hermitian operator, derivation, bicircular projection.

The third and fourth authors were partially supported by Junta de Andalucía grants FQM-3737 and FQM-194 and by MICINN project MTM 2010-17687. 
In this paper we investigate the hermitian operators on spaces of Lipschitz functions defined on a compact metric space. More precisely, for a compact metric space $(X, d)$ and a positive real parameter $\alpha \in(0,1]$, we consider the space of all $\alpha$-Lipschitz functions $f: X \rightarrow \mathbb{C}$ such that

$$
p_{\alpha}(f):=\sup \left\{\frac{|f(x)-f(y)|}{d(x, y)^{\alpha}}: x, y \in X, x \neq y\right\}<\infty,
$$

and also the subspace of all $\alpha$-Lipschitz functions $f: X \rightarrow \mathbb{C}$ satisfying the additional local flatness condition:

$$
\lim _{d(x, y) \rightarrow 0} \frac{|f(x)-f(y)|}{d(x, y)^{\alpha}}=0 .
$$

These two spaces with the standard operations of addition, multiplication and scalar multiplication are complex algebras, and when equipped with the norm

$$
\|f\|_{\alpha}=p_{\alpha}(f)+\|f\|_{\infty}
$$

become Banach algebras. These two algebras are denoted by $\operatorname{Lip}\left(X, d^{\alpha}\right)$ and $\operatorname{lip}\left(X, d^{\alpha}\right)$, respectively.

It is important to observe that $\operatorname{Lip}\left(X, d^{\alpha}\right)$ and $\operatorname{lip}\left(X, d^{\alpha}\right)$ are unital semi-simple commutative complex Banach algebras, and $\operatorname{lip}\left(X, d^{\alpha}\right)$ is a closed subalgebra of $\operatorname{Lip}\left(X, d^{\alpha}\right)$. Notice that $\operatorname{lip}(X, d)$ may contain only constant functions, for example $\operatorname{lip}[0,1]$ with the usual metric. When $X=[0,1]$ or $X=\mathbb{T}$ with the usual metrics, $\operatorname{Lip}\left(X, d^{\alpha}\right)$ and $\operatorname{lip}\left(X, d^{\alpha}\right)$ are among the classical algebras considered by de Leeuw in [4]. These algebras were first studied by Sherbert in [14,15].

In [2], it was shown that the hermitian operators on the Lipschitz spaces $\operatorname{Lip}[0,1]$ and lip $\alpha, 0<\alpha<1$, are real multiples of the identity operator. In this paper we prove that the same property holds for the spaces $\operatorname{Lip}(X, d)$ and the spaces $\operatorname{lip}\left(X, d^{\alpha}\right)$ with $0<\alpha<1$, for $(X, d)$ a compact metric space. This generalizes the aforementioned result.

We also mention the natural connection between hermitian operators and the class of bi-circular projections. A projection $P$ on a complex Banach space is bicircular if $e^{i s} P+e^{i t}(I-P)$ is an isometry for all $s, t \in \mathbb{R}$. This type of projection was introduced by Stachó and Zalar in [17. Jamison [10] showed that these projections are exactly the hermitian projections. Our result implies that the only bi-circular projections on $\operatorname{Lip}(X, d)$ and $\operatorname{lip}\left(X, d^{\alpha}\right)$ with $0<\alpha<1$ are the trivial projections, 0 and $I$.

\section{Preliminaries}

In this section we give a representation for all surjective linear isometries on $\operatorname{Lip}(X, d)$ or $\operatorname{lip}\left(X, d^{\alpha}\right)(0<\alpha<1)$ that fix the constant function everywhere equal to 1 . Then we characterize the hermitian elements of $\operatorname{Lip}(X, d)$ and $\operatorname{lip}\left(X, d^{\alpha}\right)$ $(0<\alpha<1)$. The last result provides a useful description of the continuous linear functionals on both spaces.

Throughout this paper $(X, d)$ is a compact metric space, $1_{X}$ denotes the constant function equal to 1 on $X, I_{X}$ represents the identity function on $X$ and $I$ is the identity operator on $\operatorname{Lip}(X, d)$ or $\operatorname{lip}\left(X, d^{\alpha}\right), 0<\alpha<1$. For each $x \in X, \delta_{x}$ stands for the evaluation functional at the point $x$ defined on $\operatorname{Lip}(X, d)$ or $\operatorname{lip}\left(X, d^{\alpha}\right)$, $0<\alpha<1$. 
Our approach requires that the surjective linear isometries on the spaces $\operatorname{Lip}(X, d)$ and $\operatorname{lip}\left(X, d^{\alpha}\right)(0<\alpha<1)$ have a suitable representation. Rao and Roy [13] proved that any surjective linear isometry of Lip $[0,1]$ can be expressed as a weighted composition operator $f \mapsto \tau f \circ \varphi(f \in \operatorname{Lip}[0,1])$ where $\tau$ is a scalar of modulus 1 and $\varphi$ is a surjective isometry of $[0,1]$. They asked whether every isometry on the Banach spaces $\operatorname{Lip}(X, d)$ and $\operatorname{lip}\left(X, d^{\alpha}\right)(0<\alpha<1)$ are induced by the isometries of the metric space $X$. Next we derive a characterization of surjective linear isometries on these spaces that fix $1_{X}$. This characterization follows from a theorem due to Jarosz in [11, a theorem in [8] (page 144) and a result by Sherbert in [14].

Theorem 2.1. Let $X$ be a compact metric space. Then $T: \operatorname{Lip}(X, d) \rightarrow \operatorname{Lip}(X, d)$ is a surjective linear isometry such that $T\left(1_{X}\right)=1_{X}$ if and only if there exists a surjective isometry $\varphi: X \rightarrow X$ such that $T$ is of the form $T(f)=f \circ \varphi$ for all $f \in \operatorname{Lip}(X, d)$. The same characterization holds for a surjective linear isometry $T$ on $\operatorname{lip}\left(X, d^{\alpha}\right)(0<\alpha<1)$ such that $T\left(1_{X}\right)=1_{X}$.

Proof. It is straightforward to check that an operator $T$ of the form described in the theorem is a surjective isometry. Then we just prove the reversed implication.

Let $A(X)$ represent either $\operatorname{Lip}(X, d)$ or $\operatorname{lip}\left(X, d^{\alpha}\right)$ with $0<\alpha<1$ and let $C(X)$ be the algebra of continuous complex-valued functions on $X$. We first observe that $A(X)$ is a regular subspace of $C(X)$ and the sum norm is a $p$-norm for the norm on $\mathbb{R}^{2}$ given by $p(s, t)=|s|+|t|$. Let us recall (see [1]) that given a compact Hausdorff space $X$, a complex linear subspace $A$ of $C(X)$ that contains the function $1_{X}$, is said to be regular if for any $\varepsilon>0$, any $x_{0} \in \mathrm{Ch} A$ and any open neighborhood $U$ of $x_{0}$, there is an $f \in A$ with $\|f\|_{\infty} \leq 1+\varepsilon, f\left(x_{0}\right)=1$, and $|f(x)|<\varepsilon$ for $x \in X \backslash U$. Ch $A$ denotes the set of extreme points $F$ of the unit ball of $\left(A,\|\cdot\|_{\infty}\right)$ * such that $F\left(1_{X}\right)=1$, and we identify $\operatorname{Ch} A$ with a subset of $X$. Suppose that $T$ is a surjective linear isometry on $A(X)$ such that $T\left(1_{X}\right)=1_{X}$. An application of the main theorem in [11] to $A(X)$ yields that $T$ is a surjective isometry on $\left(A(X),\|\cdot\|_{\infty}\right)$.

Next we quote a theorem from Hoffman's book [8, p. 44]: Let $X$ be a compact Hausdorff space and let $B$ be a complex linear subalgebra of $C(X)$ that contains the function $1_{X}$. Suppose that $S$ is a linear map of $B$ onto $B$ such that $\|S(f)\|_{\infty}=$ $\|f\|_{\infty}$ for all $f \in B$. If $S\left(1_{X}\right)=1_{X}$, then $S$ is multiplicative.

Therefore $T$ is an automorphism of $A(X)$. By Sherbert's theorem [14, Corollary 5.2], every automorphism $T$ of $\operatorname{Lip}(X, d)$ that carries $1_{X}$ into $1_{X}$ is of the form $T(f)=f \circ \varphi$, where $\varphi: X \rightarrow X$ is a homeomorphism. Similarly, we can prove that this is also true for those automorphisms of $\operatorname{lip}\left(X, d^{\alpha}\right)(0<\alpha<1)$ that fix $1_{X}$.

We now show that $\varphi$ is an isometry of $X$. Observe that given any $\alpha \in(0,1]$, we have $p_{\alpha}(T(f))=p_{\alpha}(f)$ for all $f \in A(X)$ since $T$ is an isometry for both norms $\|\cdot\|_{\alpha}$ and $\|\cdot\|_{\infty}$.

For the case $A(X)=\operatorname{Lip}(X, d)$, fix $y \in X$ and define $f_{y}: X \rightarrow \mathbb{R}$ by $f_{y}(z)=$ $d(z, \varphi(y))$ for all $z \in X$. Clearly, $f_{y} \in \operatorname{Lip}(X, d)$ and $p_{1}\left(f_{y}\right) \leq 1$. For any $x, y \in X$, we have

$$
\begin{aligned}
d(\varphi(x), \varphi(y)) & =\left|f_{y}(\varphi(x))-f_{y}(\varphi(y))\right| \\
& =\left|T\left(f_{y}\right)(x)-T\left(f_{y}\right)(y)\right| \\
& \leq p_{1}\left(T\left(f_{y}\right)\right) d(x, y) \\
& \leq d(x, y) .
\end{aligned}
$$


For the case $A(X)=\operatorname{lip}\left(X, d^{\alpha}\right)(0<\alpha<1)$, fix $x, y \in X, x \neq y$, choose $\beta \in(\alpha, 1)$ and define

$$
f_{x y}(z)=\frac{d(z, \varphi(y))^{\beta}-d(z, \varphi(x))^{\beta}}{2 d(\varphi(x), \varphi(y))^{\beta-\alpha}}, \quad \forall z \in X .
$$

It is not hard to check that $f_{x y} \in \operatorname{lip}\left(X, d^{\alpha}\right)$ and $p_{\alpha}\left(f_{x y}\right)=1$ (see, for example, [12, p. 62]). An easy calculation gives

$$
\begin{aligned}
d(\varphi(x), \varphi(y))^{\alpha} & =\left|f_{x y}(\varphi(x))-f_{x y}(\varphi(y))\right| \\
& =\left|T\left(f_{x y}\right)(x)-T\left(f_{x y}\right)(y)\right| \\
& \leq p_{\alpha}\left(T\left(f_{x y}\right)\right) d(x, y)^{\alpha} \\
& =d(x, y)^{\alpha} .
\end{aligned}
$$

In either case we have $d(\varphi(x), \varphi(y)) \leq d(x, y)$ for all $x, y \in X$.

Since $T^{-1}$ is also a surjective linear isometry on $A(X)$ such that $T^{-1}\left(1_{X}\right)=1_{X}$, the same argument used above implies the existence of a homeomorphism $\phi: X \rightarrow$ $X$ such that $T^{-1}(f)=f \circ \phi$ for all $f \in A(X)$. Therefore $d(\phi(x), \phi(y)) \leq d(x, y)$ for all $x, y \in X$. Given $x \in X$, we have

$$
f\left(\varphi^{-1}(x)\right)=T\left(T^{-1}(f)\right)\left(\varphi^{-1}(x)\right)=T^{-1}(f)(x)=f(\phi(x))
$$

for all $f \in A(X)$. Since $A(X)$ separates the points of $X$, this implies that $\varphi^{-1}=\phi$. Consequently, $\varphi$ is a surjective isometry. This completes the proof of the theorem.

We will next characterize the hermitian elements of the spaces $\operatorname{Lip}(X, d)$ and $\operatorname{lip}\left(X, d^{\alpha}\right), 0<\alpha<1$.

Lemma 2.2. Let $(X, d)$ be a compact metric space and $h \in \operatorname{Lip}(X, d)$ (or $\operatorname{lip}\left(X, d^{\alpha}\right)$, $0<\alpha<1)$. Then $h$ is a hermitian element in $\operatorname{Lip}(X, d)\left(\right.$ or $\left.\operatorname{lip}\left(X, d^{\alpha}\right)\right)$ if and only if $h$ is a real constant function.

Proof. Assume that $h$ is hermitian in $\operatorname{Lip}(X, d)$. Then $F(h) \in V(h) \subset \mathbb{R}$ for all $F \in \operatorname{Lip}(X, d)^{*}$ such that $\|F\|=F\left(1_{X}\right)=1$. In particular, $h(x)=\delta_{x}(h) \in \mathbb{R}$ for all $x \in X$, and so $h$ is real-valued. Using that $\left|e^{a}-e^{b}\right| \leq|a-b| \exp (\max \{|a|,|b|\})$ for all $a, b \in \mathbb{C}$, we deduce that $\exp (i h)$ is a function in $\operatorname{Lip}(X, d)$. We also have that, for each $t \in \mathbb{R},\|\exp (i t h)\|_{1}=1$. Since $\|\exp (i t h)\|_{\infty}=1$, it follows that $p_{1}(\exp (i t h))=0$. Hence $\exp (i t h)$ is a constant function on $X$ for all $t \in \mathbb{R}$ which implies that $h$ is constant.

Conversely, if $h$ is a real constant function, then $h$ is a real multiple of $1_{X}$. Therefore $h$ is hermitian in $\operatorname{Lip}(X, d)$. The same proof works for $\operatorname{lip}\left(X, d^{\alpha}\right), 0<$ $\alpha<1$.

Following an idea of de Leeuw [4], we embed the Banach spaces $\operatorname{Lip}(X, d)$ and $\operatorname{lip}\left(X, d^{\alpha}\right)(0<\alpha<1)$ isometrically into some suitable spaces of complex-valued continuous functions.

Let $X$ be a compact metric space and let $\widetilde{X}$ be the set $\left\{(x, y) \in X^{2}: x \neq y\right\}$. It is easy to check that $\widetilde{X}$ is completely regular; we denote by $\beta \widetilde{X}$ the Stone-Čech compactification of $\widetilde{X}$. Let $C(X \cup \beta \widetilde{X})$ denote the Banach space of all complexvalued continuous functions on $X \cup \beta \widetilde{X}$, under the norm

$$
\|f\|=\left\|\left.f\right|_{X}\right\|_{\infty}+\left\|\left.f\right|_{\beta \widetilde{X}}\right\|_{\infty} \quad(f \in C(X \cup \beta \tilde{X})),
$$


and let $C_{0}(X \cup \tilde{X})$ denote the Banach space of all complex-valued continuous functions on $X \cup \widetilde{X}$ vanishing at infinity, endowed with the norm

$$
\|f\|=\left\|\left.f\right|_{X}\right\|_{\infty}+\left\|\left.f\right|_{\widetilde{X}}\right\|_{\infty} \quad\left(f \in C_{0}(X \cup \widetilde{X})\right) .
$$

We now recall that the Riesz representation theorem states that the map $\mu \mapsto F_{\mu}$, given by

$$
F_{\mu}(f)=\int_{X \cup \beta \widetilde{X}} f d \mu \quad(f \in C(X \cup \beta \widetilde{X})),
$$

defines an isometric isomorphism from the Banach space $\mathcal{M}(X \cup \beta \widetilde{X})$ of all complexvalued regular Borel measures on $X \cup \beta \widetilde{X}$ equipped with the norm of total variation:

$$
\|\mu\|=|\mu|(X \cup \beta \widetilde{X}) \quad(\mu \in \mathcal{M}(X \cup \beta \widetilde{X}))
$$

onto the dual space of $\left(C(X \cup \beta \widetilde{X}),\|\cdot\|_{\infty}\right)$. Similarly, the map $\nu \mapsto G_{\nu}$ defined by

$$
G_{\nu}(f)=\int_{X \cup \widetilde{X}} f d \nu \quad\left(f \in C_{0}(X \cup \widetilde{X})\right)
$$

is an isometric isomorphism from the Banach space $\mathcal{M}(X \cup \tilde{X})$ with the norm

$$
\|\nu\|=|\nu|(X \cup \tilde{X}) \quad(\nu \in \mathcal{M}(X \cup \tilde{X}))
$$

onto the dual space of $\left(C_{0}(X \cup \widetilde{X}),\|\cdot\|_{\infty}\right)$.

For each $f \in \operatorname{Lip}(X, d)$ or $f \in \operatorname{lip}\left(X, d^{\alpha}\right), 0<\alpha<1$, we set $\tilde{f}: \widetilde{X} \rightarrow \mathbb{C}$ to be the map given by

$$
\widetilde{f}(x, y)=\frac{f(x)-f(y)}{d(x, y)^{\alpha}}, \quad \forall(x, y) \in \widetilde{X},
$$

where $\alpha=1$ when $f \in \operatorname{Lip}(X, d)$. It is easy to show that $\tilde{f}$ is continuous on $\tilde{X}$ and $\|\tilde{f}\|_{\infty}=p_{\alpha}(f)(0<\alpha \leq 1)$. Hence there exists a unique continuous function $\beta \tilde{f}$ on $\beta \widetilde{X}$ such that $\left.(\beta \widetilde{f})\right|_{\tilde{X}}=\widetilde{f}$ and $\|\beta \widetilde{f}\|_{\infty}=\|\widetilde{f}\|_{\infty}$. Furthermore, if $f \in \operatorname{lip}\left(X, d^{\alpha}\right)$, then $\tilde{f}$ vanishes at infinity on $\tilde{X}$. The maps $\Phi: \operatorname{Lip}(X, d) \rightarrow C(X \cup \beta \widetilde{X})$ and $\Psi: \operatorname{lip}\left(X, d^{\alpha}\right) \rightarrow C_{0}(X \cup \widetilde{X})$, defined by

$$
\Phi(f)(w)= \begin{cases}f(w) & \text { if } w \in X, \\ \beta \widetilde{f}(w) & \text { if } w \in \beta \widetilde{X},\end{cases}
$$

and

$$
\Psi(f)(w)= \begin{cases}f(w) & \text { if } w \in X, \\ \widetilde{f}(w) & \text { if } w \in \tilde{X},\end{cases}
$$

are isometric linear embeddings from $\operatorname{Lip}(X, d)$ with the norm $\|\cdot\|_{1}$ into $C(X \cup \beta \widetilde{X})$, and from $\operatorname{lip}\left(X, d^{\alpha}\right)$ with the norm $\|\cdot\|_{\alpha}$ into $C_{0}(X \cup \widetilde{X})$, respectively.

The Hahn-Banach theorem and the Riesz representation theorem yield the following lemma.

Lemma 2.3. Let $(X, d)$ be a compact metric space. 
(1) For each $F \in \operatorname{Lip}(X, d)^{*}$, there exists $\mu \in \mathcal{M}(X \cup \beta \tilde{X})$ with $\|F\| \leq\|\mu\|$ satisfying

$$
F(f)=\int_{X \cup \beta \widetilde{X}} \Phi(f)(w) d \mu(w), \quad \forall f \in \operatorname{Lip}(X, d) .
$$

(2) Let $\alpha \in(0,1)$. For each $G \in \operatorname{lip}\left(X, d^{\alpha}\right)^{*}$, there exists $\nu \in \mathcal{M}(X \cup \widetilde{X})$ with $\|G\| \leq\|\nu\|$ such that

$$
G(f)=\int_{X \cup \tilde{X}} \Psi(f)(w) d \nu(w), \quad \forall f \in \operatorname{lip}\left(X, d^{\alpha}\right) .
$$

Proof. Let $F \in \operatorname{Lip}(X, d)^{*}$. The functional $T: \Phi(\operatorname{Lip}(X, d)) \rightarrow \mathbb{C}$, defined by $T(\Phi(f))=F(f)$ for all $f \in \operatorname{Lip}(X, d)$, is linear, continuous and $\|T\|=\|F\|$. By the Hahn-Banach theorem, there exists a linear continuous functional $\widetilde{T}: C(X \cup \beta \widetilde{X}) \rightarrow$ $\mathbb{C}$ such that $\widetilde{T}(\Phi(f))=T(\Phi(f))$ for all $f \in \operatorname{Lip}(X, d)$ and $\|\widetilde{T}\|=\|T\|$.

Since $\|g\| \leq 2\|g\|_{\infty}$ for all $g \in C(X \cup \beta \widetilde{X})$, it follows that the linear functional $\widetilde{T}$ is continuous on the space $C(X \cup \beta \widetilde{X})$ equipped with the norm $\|\cdot\|_{\infty}$. We denote by $\|\cdot\|_{\infty}^{*}$ the norm on the dual Banach space of $\left(C(X \cup \beta \widetilde{X}),\|\cdot\|_{\infty}\right)$. By the Riesz representation theorem, there exists $\mu \in \mathcal{M}(X \cup \beta \widetilde{X})$ with $\|\widetilde{T}\|_{\infty}^{*}=\|\mu\|$ satisfying

$$
\widetilde{T}(g)=\int_{X \cup \beta \widetilde{X}} g(w) d \mu(w), \quad \forall g \in C(X \cup \beta \widetilde{X}) .
$$

Since $\|g\|_{\infty} \leq\|g\|$ for all $g \in C(X \cup \beta \widetilde{X})$, we have $\|\widetilde{T}\| \leq\|\widetilde{T}\|_{\infty}^{*}$, and so $\|F\| \leq\|\mu\|$. Moreover,

$$
F(f)=T(\Phi(f))=\widetilde{T}(\Phi(f))=\int_{X \cup \beta \widetilde{X}} \Phi(f)(w) d \mu(w)
$$

for all $f \in \operatorname{Lip}(X, d)$, as we wanted. Similarly, we prove statement (2).

Such a $\mu$ is called a representing measure for $F$ (analogously, $\nu$ for $G$ ). We should note that a representing measure for $F$ or $G$ is not always determined uniquely.

\section{The MAIN RESUlT}

In this section we describe all the hermitian operators on $\operatorname{Lip}(X, d)$ or $\operatorname{lip}\left(X, d^{\alpha}\right)$ with $0<\alpha<1$. We proceed with the statement and proof of our main result.

Theorem 3.1. Let $(X, d)$ be a compact metric space. A bounded linear operator $T: \operatorname{Lip}(X, d) \rightarrow \operatorname{Lip}(X, d)$ is hermitian if and only if $T$ is a real multiple of the identity operator on $\operatorname{Lip}(X, d)$. An analogous assertion holds for $T: \operatorname{lip}\left(X, d^{\alpha}\right) \rightarrow$ $\operatorname{lip}\left(X, d^{\alpha}\right)$ with $0<\alpha<1$.

Before proving this theorem we set notation and prove some preliminary lemmas. Let $A(X)$ denote either $\operatorname{Lip}(X, d)$ or $\operatorname{lip}\left(X, d^{\alpha}\right), 0<\alpha<1$. Recall that $\alpha=1$ in the case $A(X)=\operatorname{Lip}(X, d)$.

Lemma 3.2. If $T: A(X) \rightarrow A(X)$ is a hermitian bounded linear operator, then the following statements hold:

(i) There exists $\lambda \in \mathbb{R}$ such that $T\left(1_{X}\right)=\lambda 1_{X}$.

(ii) For each $t \in \mathbb{R}, \exp (i t(T-\lambda I))$ is a surjective linear isometry on $A(X)$ fixing $1_{X}$. 
(iii) For each $t \in \mathbb{R}$, there exists a surjective isometry $\varphi_{t}$ on $X$ such that

$$
\exp (i t(T-\lambda I))(f)(x)=f\left(\varphi_{t}(x)\right), \quad \forall f \in A(X), \forall x \in X .
$$

(iv) $\left\{\varphi_{t}\right\}_{t \in \mathbb{R}}$ is a one-parameter group of surjective isometries on $X$ such that, for each $x \in X$, the map $t \mapsto \varphi_{t}(x)$ from $\mathbb{R}$ to $X$ is continuous.

(v) For every $f \in A(X)$,

$$
\lim _{t \rightarrow 0}\left(f \circ \varphi_{t}-f\right)(x)=0, \quad \forall x \in X,
$$

and

$$
\lim _{t \rightarrow 0} \frac{\left(f \circ \varphi_{t}-f\right)(x)-\left(f \circ \varphi_{t}-f\right)(y)}{d(x, y)^{\alpha}}=0, \quad \forall(x, y) \in \widetilde{X} .
$$

Proof. (i) For each $F \in A(X)^{*}$ with $\|F\|=F\left(1_{X}\right)=1$, define $\Phi_{F}: \mathcal{B}(A(X)) \rightarrow \mathbb{C}$ by

$$
\Phi_{F}(S)=F\left(S\left(1_{X}\right)\right), \quad \forall S \in \mathcal{B}(A(X)) .
$$

It is easy to check that $\Phi_{F}$ is a linear functional on $\mathcal{B}(A(X))$, and since

$$
\left|\Phi_{F}(S)\right|=\left|F\left(S\left(1_{X}\right)\right)\right| \leq\|F\|\left\|S\left(1_{X}\right)\right\|_{\alpha} \leq\|S\|\left\|1_{X}\right\|_{\alpha}=\|S\|,
$$

for all $S \in \mathcal{B}(A(X))$, then $\Phi_{F}$ is continuous and $\left\|\Phi_{F}\right\| \leq 1$. Moreover, $\Phi_{F}(I)=$ $F\left(1_{X}\right)=1$; hence $\left\|\Phi_{F}\right\| \geq\left|\Phi_{F}(I)\right|=1$ and thus $\left\|\Phi_{F}\right\|=\Phi_{F}(I)=1$.

Since $T \in \mathcal{B}(A(X))$ is hermitian, it follows that $F\left(T\left(1_{X}\right)\right)=\Phi_{F}(T) \in V(T) \subset \mathbb{R}$ for all $F \in A(X)^{*}$ such that $\|F\|=F\left(1_{X}\right)=1$. This means that $T\left(1_{X}\right)$ is a hermitian element in $A(X)$. Then, according to Lemma 2.2 , there exists $\lambda \in \mathbb{R}$ such that $T\left(1_{X}\right)=\lambda 1_{X}$.

(ii) By (i), we have $(T-\lambda I)\left(1_{X}\right)=0$ and so $\exp (i t(T-\lambda I))\left(1_{X}\right)=1_{X}$ for all $t \in \mathbb{R}$. Indeed, since

$$
\exp (i t(T-\lambda I))=I+\sum_{n=1}^{\infty} i^{n} t^{n}(T-\lambda I)^{n},
$$

it follows that

$$
\exp (i t(T-\lambda I))\left(1_{X}\right)=1_{X}+\sum_{n=1}^{\infty} i^{n} t^{n}(T-\lambda I)^{n}\left(1_{X}\right)=1_{X} .
$$

Since $T$ and $\lambda I$ are hermitian operators in $\mathcal{B}(A(X))$, it is easily seen that so is $T-\lambda I$. Indeed, using the fact that $\exp (i t(T-\lambda I))=\exp (i t T) \exp (-i t \lambda I)$ for all $t \in \mathbb{R}$, we have

$$
\begin{aligned}
1=\left\|1_{X}\right\|_{\alpha} & =\left\|\exp (i t(T-\lambda I))\left(1_{X}\right)\right\|_{\alpha} \leq\|\exp (i t(T-\lambda I))\| \\
& \leq\|\exp (i t T)\|\|\exp (-i t \lambda I)\|=1 .
\end{aligned}
$$

Therefore, for each $t \in \mathbb{R}, \exp (i t(T-\lambda I))$ is a linear isometry from $A(X)$ onto itself, fixing $1_{X}$.

(iii) In view of (ii), by applying Theorem 2.1, for each $t \in \mathbb{R}$ there exists a surjective isometry $\varphi_{t}$ on $X$ such that

$$
\exp (i t(T-\lambda I))(f)(x)=f\left(\varphi_{t}(x)\right), \quad \forall f \in A(X), \forall x \in X .
$$


(iv) Using the fact that $A(X)$ separates the points of $X$, it is easily derived that $\varphi_{(s+t)}=\varphi_{s} \circ \varphi_{t}$ for all $s, t \in \mathbb{R}$ and $\varphi_{0}=I_{X}$. More precisely, given $f \in A(X)$ and $x \in X$, we have

$$
\begin{aligned}
f\left(\varphi_{s+t}(x)\right) & =\exp (i(s+t)(T-\lambda I))(f)(x) \\
& =\exp (i(t+s)(T-\lambda I))(f)(x) \\
& =\exp (i t(T-\lambda I)) \exp (i s(T-\lambda I))(f)(x) \\
& =\exp (i t(T-\lambda I))(\exp (i s(T-\lambda I))(f))(x) \\
& =\exp (i s(T-\lambda I))(f)\left(\varphi_{t}(x)\right) \\
& =f\left(\varphi_{s}\left(\varphi_{t}(x)\right)\right) \\
& =f\left(\varphi_{s} \circ \varphi_{t}(x)\right)
\end{aligned}
$$

and

$$
f\left(\varphi_{0}(x)\right)=\exp (i 0(T-\lambda I))(f)(x)=\exp (0)(f)(x)=I(f)(x)=f(x) .
$$

We next prove that for each $x \in X$, the map $t \mapsto \varphi_{t}(x)$ from $\mathbb{R}$ to $X$ is continuous. Note first that $\delta: X \rightarrow A(X)^{*}$ defined by $\delta(x)=\delta_{x}$ is a Lipschitz bijection from $\left(X, d^{\alpha}\right)$ onto $\delta(X)$. Indeed, $\delta$ is injective since $A(X)$ separates points; and given $x, y \in X$, we have

$$
|(\delta(x)-\delta(y))(f)|=|f(x)-f(y)| \leq\|f\|_{\alpha} d(x, y)^{\alpha}
$$

for all $f \in A(X)$. Hence $\|\delta(x)-\delta(y)\| \leq d(x, y)^{\alpha}$. Since $X$ is compact, we deduce that $\delta^{-1}: \delta(X) \rightarrow X$ is continuous. Notice that $\delta^{-1}\left(\delta_{x}\right)=x$ for all $x \in X$.

Fix $x \in X$. The maps $t \mapsto \exp (i t(T-\lambda I))$ from $\mathbb{R}$ to $\mathcal{B}(A(X)), U \mapsto U^{*}$ from $\mathcal{B}(A(X))$ to $\mathcal{B}\left(A(X)^{*}\right)$ and $S \mapsto S(\delta(x))$ from $\mathcal{B}\left(A(X)^{*}\right)$ to $A(X)^{*}$ are clearly continuous. From (5), we deduce that

$$
(\exp (i t(T-\lambda I)))^{*}(\delta(x))=\delta\left(\varphi_{t}(x)\right) \quad(t \in \mathbb{R}, x \in X) .
$$

Since

$$
\varphi_{t}(x)=\delta^{-1}(\exp (i t(T-\lambda I)))^{*}(\delta(x)) \quad(t \in \mathbb{R}, x \in X),
$$

we conclude that $t \mapsto \varphi_{t}(x)$ from $\mathbb{R}$ to $X$ is continuous.

(v) Let $f \in A(X)$. Given $x \in X$, we have $\lim _{t \rightarrow 0}\left(f \circ \varphi_{t}\right)(x)=\left(f \circ \varphi_{0}\right)(x)=f(x)$ by (iv), and thus $\lim _{t \rightarrow 0}\left(f \circ \varphi_{t}-f\right)(x)=0$. Using this, for $(x, y) \in \widetilde{X}$, we deduce that

$$
\lim _{t \rightarrow 0} \frac{\left(f \circ \varphi_{t}-f\right)(x)-\left(f \circ \varphi_{t}-f\right)(y)}{d(x, y)^{\alpha}}=0 .
$$

We recall that for $f \in A(X)$ the map $\tilde{f}: \widetilde{X} \rightarrow \mathbb{C}$ is defined to be

$$
\widetilde{f}(x, y)=(f(x)-f(y)) / d(x, y)^{\alpha} .
$$

We recall that $\beta \tilde{X}$ represents the Stone-Čech compactification of $\tilde{X}$. This entails that every bounded, continuous and scalar-valued map defined on $\widetilde{X}$ has a unique continuous extension to $\beta \widetilde{X}$.

Lemma 3.3. If $f \in A(X)$, then

$$
\lim _{t \rightarrow 0} \beta \widetilde{f}_{t}(w)=0, \quad \forall w \in \beta \widetilde{X},
$$

where, for each $t \in \mathbb{R}, f_{t}$ denotes the function $f \circ \varphi_{t}-f$. 
Proof. We define $g:[-1,1] \times \widetilde{X} \rightarrow \mathbb{C}$ by

$$
g(t,(x, y))=\frac{\left(f \circ \varphi_{t}-f\right)(x)-\left(f \circ \varphi_{t}-f\right)(y)}{d(x, y)^{\alpha}} .
$$

The function $g$ is continuous and bounded. In fact, we have

$$
|g(t,(x, y))| \leq p_{\alpha}(f)+p_{\alpha}(f)=2 p_{\alpha}(f)
$$

for all $t \in[-1,1]$ and $(x, y) \in \widetilde{X}$. For the continuity of $\mathrm{g}$, define $\sigma:[-1,1] \times \widetilde{X} \rightarrow$ $A(X)^{*}$ by

$$
\sigma(t,(x, y))=\frac{\delta\left(\varphi_{t}(x)\right)-\delta(x)-\left(\delta\left(\varphi_{t}(y)\right)-\delta(y)\right)}{d(x, y)^{\alpha}}
$$

and notice that

$$
g(t,(x, y))=\sigma(t,(x, y))(f) \quad(t \in[-1,1],(x, y) \in \tilde{X}) .
$$

Taking into account the equality ([6]), for any $t, s \in[-1,1]$ and $x, y \in X$, we have

$$
\begin{aligned}
\left\|\delta\left(\varphi_{t}(x)\right)-\delta\left(\varphi_{s}(y)\right)\right\| & =\left\|(\exp (i t(T-\lambda I)))^{*}(\delta(x))-(\exp (i s(T-\lambda I)))^{*}(\delta(y))\right\| \\
& \leq\left\|(\exp (i t(T-\lambda I)))^{*}\right\|\|\delta(x)-\delta(y)\| \\
& +\left\|(\exp (i t(T-\lambda I)))^{*}-(\exp (i s(T-\lambda I)))^{*}\right\|\|\delta(y)\| \\
& \leq d(x, y)+\|\exp (i t(T-\lambda I))-\exp (i s(T-\lambda I))\|\|\delta(y)\| .
\end{aligned}
$$

Let us recall now that if $A$ and $B$ are bounded commuting operators on a Banach algebra, then

$$
\|\exp (i A)-\exp (i B)\| \leq\|A-B\| \exp (\max \{\|A\|,\|B\|\}) .
$$

Applying this formula to $A=t(T-\lambda I)$ and $B=s(T-\lambda I)$, we obtain

$$
\|\exp (i t(T-\lambda I))-\exp (i s(T-\lambda I))\| \leq|t-s| k,
$$

where $k=\|T-\lambda I\| \exp (\|T-\lambda I\|)$ is a constant, and so

$$
\left\|\delta\left(\varphi_{t}(x)\right)-\delta\left(\varphi_{s}(y)\right)\right\| \leq d(x, y)^{\alpha}+k|t-s| .
$$

Therefore, for every $t, s \in[-1,1]$ and $x, y \in X$, we have

$$
\left\|\delta\left(\varphi_{t}(x)\right)-\delta(x)-\left(\delta\left(\varphi_{s}(y)\right)-\delta(y)\right)\right\| \leq 2 d(x, y)^{\alpha}+k|t-s| .
$$

Hence the mapping $(t,(x, y)) \mapsto \delta\left(\varphi_{t}(x)\right)-\delta(x)$, defined on $[-1,1] \times \widetilde{X}$ and with values in $A(X)^{*}$, is continuous. Since $(x, y) \mapsto d(x, y)^{\alpha}$ from $\widetilde{X}$ to $\mathbb{R}$ is continuous, it follows that $\sigma$ is continuous. Hence, given $\varepsilon>0$ and $\left(t_{0},\left(x_{0}, y_{0}\right)\right) \in[-1,1] \times$ $\widetilde{X}$, there is a neighborhood $V$ of $\left(t_{0},\left(x_{0}, y_{0}\right)\right)$ such that if $(t,(x, y)) \in V$, then $\left\|\sigma(t,(x, y))-\sigma\left(t_{0},\left(x_{0}, y_{0}\right)\right)\right\|<\varepsilon /\left(1+\|f\|_{\alpha}\right)$. Therefore, for every $(t,(x, y)) \in V$, we have

$$
\left|g(t,(x, y))-g\left(t_{0},\left(x_{0}, y_{0}\right)\right)\right|<\frac{\varepsilon}{1+\|f\|_{\alpha}}\|f\|_{\alpha}<\varepsilon,
$$

and this proves that $g$ is continuous. 
We now check that the mapping $t \mapsto g(t, \cdot)$ from $[-1,1]$ into $C(\widetilde{X})$ is continuous. Indeed, by using (6) and (8) we have

$$
\begin{aligned}
\mid g(t, & (x, y))-g(s,(x, y)) \mid \\
& =\left|\frac{\delta\left(\varphi_{t}(x)\right)-\delta\left(\varphi_{t}(y)\right)-\left(\delta\left(\varphi_{s}(x)\right)-\delta\left(\varphi_{s}(y)\right)\right)}{d(x, y)^{\alpha}}(f)\right| \\
& \leq\left\|\frac{\left((\exp (i t(T-\lambda I)))^{*}-(\exp (i s(T-\lambda I)))^{*}\right)(\delta(x)-\delta(y))}{d(x, y)^{\alpha}}\right\|\|f\|_{\alpha} \\
& \leq k\|f\|_{\alpha}|t-s|
\end{aligned}
$$

for all $t, s \in[-1,1]$ and $(x, y) \in \tilde{X}$, and so $\|g(t, \cdot)-g(s, \cdot)\|_{\infty} \leq k\|f\|_{\alpha}|t-s|$.

Then $g$ has a continuous extension $h$ from $[-1,1] \times \beta \widetilde{X}$ to $\mathbb{C}$ by [7, Lemma 2]. Hence $h(0,(x, y))=g(0,(x, y))=0$ for all $(x, y) \in \widetilde{X}$. Now fix $w \in \beta \widetilde{X}$ and take a net $\left\{\left(x_{i}, y_{i}\right)\right\}$ in $\widetilde{X}$ converging to $w$. The continuity of $h$ yields $h(0, w)=$ $\lim _{i} h\left(0,\left(x_{i}, y_{i}\right)\right)=0$. Using the continuity of $\beta \widetilde{f}_{t}$, with $t \in[-1,1]$, and the continuity of $h$, we have

$$
\begin{aligned}
\lim _{t \rightarrow 0} \beta \widetilde{f}_{t}(w) & =\lim _{t \rightarrow 0} \lim _{i} \beta \widetilde{f}_{t}\left(x_{i}, y_{i}\right)=\lim _{t \rightarrow 0} \lim _{i} \widetilde{f}_{t}\left(x_{i}, y_{i}\right)=\lim _{t \rightarrow 0} \lim _{i} g\left(t,\left(x_{i}, y_{i}\right)\right) \\
& =\lim _{t \rightarrow 0} \lim _{i} h\left(t,\left(x_{i}, y_{i}\right)\right)=\lim _{t \rightarrow 0} h(t, w)=h(0, w)=0 .
\end{aligned}
$$

This completes the proof.

We now proceed with the details for the proof of our main theorem.

Proof of Theorem 3.1. Assume that $T: A(X) \rightarrow A(X)$ is a hermitian bounded linear operator. In view of (3), (4) and (7), the maps defined in (11) and (2) satisfy

$$
\lim _{t \rightarrow 0} \Phi\left(f \circ \varphi_{t}-f\right)(w)=0, \quad \forall w \in X \cup \beta \widetilde{X},
$$

and

$$
\lim _{t \rightarrow 0} \Psi\left(f \circ \varphi_{t}-f\right)(w)=0, \quad \forall w \in X \cup \widetilde{X} .
$$

Note also that

$$
\begin{aligned}
&\left|\Phi\left(f \circ \varphi_{t}-f\right)(w)\right| \leq\left\|\Phi\left(f \circ \varphi_{t}-f\right)\right\|=\left\|f \circ \varphi_{t}-f\right\|_{1} \leq 2\|f\|_{1}, \\
& \forall t \in \mathbb{R}, \forall w \in X \cup \beta \widetilde{X},
\end{aligned}
$$

and

$$
\begin{aligned}
\left|\Psi\left(f \circ \varphi_{t}-f\right)(w)\right| \leq\left\|\Psi\left(f \circ \varphi_{t}-f\right)\right\|=\left\|f \circ \varphi_{t}-f\right\|_{\alpha} & \leq 2\|f\|_{\alpha}, \\
\forall t & \in \mathbb{R}, \forall w \in X \cup \widetilde{X} .
\end{aligned}
$$

Now, for each $t \in \mathbb{R}$, define $T_{t}: A(X) \rightarrow A(X)$ by

$$
T_{t}(f)(x)=f\left(\varphi_{t}(x)\right), \quad \forall f \in A(X), \forall x \in X .
$$

Each $T_{t}$ is an isometric algebra automorphism of $A(X)$. Moreover, it is immediate that $T_{(s+t)}=T_{s} \circ T_{t}$ for all $s, t \in \mathbb{R}$ and $T_{0}=I$. Indeed, for any $f \in A(X)$, we have $T_{(s+t)}(f)=f \circ \varphi_{(s+t)}=f \circ \varphi_{(t+s)}=f \circ \varphi_{t} \circ \varphi_{s}=T_{s}\left(f \circ \varphi_{t}\right)=T_{s}\left(T_{t}(f)\right)=T_{s} \circ T_{t}(f)$ and

$$
T_{0}(f)=f \circ \varphi_{0}=f \circ I_{X}=f .
$$


Thus $\left\{T_{t}: t \in \mathbb{R}\right\}$ is a one-parameter group of isometric algebra automorphisms of $A(X)$. Indeed, notice that $T_{(-t)}=\left(T_{t}\right)^{-1}$ for every $t \in \mathbb{R}$ since

$$
I=T_{0}=T_{(t+(-t))}=T_{t} \circ T_{(-t)}, \quad I=T_{0}=T_{((-t)+t)}=T_{(-t)} \circ T_{t} \quad(t \in \mathbb{R}) .
$$

Next we will prove that $\left\{T_{t}: t \in \mathbb{R}\right\}$ is strongly continuous; that is, the maps $t \mapsto T_{t}(f)$ from $\mathbb{R}$ to $A(X)$ are continuous for each $f \in A(X)$ [5, Chapter I, 1.1 Definition]. According to [5, Chapter I, 1.6 Theorem], it is sufficient to show that $\left\{T_{t}: t \in \mathbb{R}\right\}$ is weakly continuous; that is, the maps $t \mapsto F\left(T_{t}(f)\right)$ from $\mathbb{R}$ to $\mathbb{C}$ are continuous for every $f \in A(X)$ and $F \in A(X)^{*}$. To prove this, notice that we only need to see that the aforementioned maps are continuous at 0 , and it is easily checked.

Let $f \in \operatorname{Lip}(X, d)$ and $F \in \operatorname{Lip}(X, d)^{*}$. By Lemma 2.3 (1), there exists $\mu \in$ $\mathcal{M}(X \cup \beta \widetilde{X})$ such that

$$
F(g)=\int_{X \cup \beta \widetilde{X}} \Phi(g)(w) d \mu(w) \quad(g \in \operatorname{Lip}(X, d)) .
$$

In particular, we have

$$
F\left(T_{t}(f)-f\right)=\int_{X \cup \beta \widetilde{X}} \Phi\left(T_{t}(f)-f\right)(w) d \mu(w) \quad(t \in \mathbb{R}) .
$$

Taking into account (9) and (11), we can apply Lebesgue's bounded convergence theorem and get

$$
\begin{aligned}
\lim _{t \rightarrow 0} F\left(T_{t}(f)-f\right) & =\lim _{t \rightarrow 0} \int_{X \cup \beta \widetilde{X}} \Phi\left(T_{t}(f)-f\right)(w) d \mu(w) \\
& =\lim _{t \rightarrow 0} \int_{X \cup \beta \widetilde{X}} \Phi\left(f \circ \varphi_{t}-f\right)(w) d \mu(w) \\
& =\int_{X \cup \beta \widetilde{X}} \lim _{t \rightarrow 0} \Phi\left(f \circ \varphi_{t}-f\right)(w) d \mu(w)=0 .
\end{aligned}
$$

Similarly, for any $f \in \operatorname{lip}\left(X, d^{\alpha}\right)$ and $G \in \operatorname{lip}\left(X, d^{\alpha}\right)^{*}$, we obtain

$$
\lim _{t \rightarrow 0} G\left(T_{t}(f)-f\right)=0
$$

by using Lemma 2.3 (2) and the equalities (10) and (12).

Let $A: D(A) \subset A(X) \rightarrow A(X)$ be the generator of $\left\{T_{t}: t \in \mathbb{R}\right\}$. That is, $A$ is the linear operator

defined for every $f$ in its domain

$$
A(f)=\lim _{t \rightarrow 0} \frac{T_{t}(f)-f}{t}
$$

$$
D(A)=\left\{f \in A(X): \exists \lim _{t \rightarrow 0} \frac{T_{t}(f)-f}{t}\right\}
$$

(see [5, Chapter II, 1.2 Definition]). By the equalities (5) and (13), we have

$$
T_{t}(f)=\exp (i t(T-\lambda I))(f), \quad \forall f \in A(X) .
$$

It follows that

$$
\lim _{t \rightarrow 0} \frac{T_{t}(f)-f}{t}=i(T-\lambda I)(f), \quad \forall f \in A(X)
$$

[5. Chapter I, 2.11 Proposition]; hence $D(A)=A(X)$ and

$$
A(f)=i(T-\lambda I)(f), \quad \forall f \in A(X) .
$$


Since $\left\{T_{t}: t \in \mathbb{R}\right\}$ is a group of algebra homomorphisms on $A(X)$, then $A$ is a derivation on $A(X)$; that is, $A(f g)=A(f) g+f A(g)$ for all $f, g \in A(X)$. Indeed,

$$
\begin{aligned}
A(f g) & =\lim _{t \rightarrow 0} \frac{T_{t}(f g)-f g}{t} \\
& =\lim _{t \rightarrow 0} \frac{T_{t}(f) T_{t}(g)-f g}{t} \\
& =\lim _{t \rightarrow 0} \frac{\left(T_{t}(f)-f\right) T_{t}(g)+f\left(T_{t}(g)-g\right)}{t} \\
& =\lim _{t \rightarrow 0}\left(\frac{T_{t}(f)-f}{t} T_{t}(g)+f \frac{T_{t}(g)-g}{t}\right) \\
& =A(f) T_{0}(g)+f A(g) \\
& =A(f) g+f A(g) .
\end{aligned}
$$

Since all linear derivations on a complex commutative semi-simple Banach algebra are trivial [16, 18, it follows that $A$ is zero, and so $T=\lambda I$, as desired. The converse implication follows immediately, and so the proof of the theorem is finished.

\section{ACKNOWLEDGEMENT}

The authors wish to thank the referee for making several suggestions which improved this paper.

\section{REFERENCES}

[1] E. Berkson and H. Porta, Hermitian operators and one-parameter groups of isometries in Hardy spaces, Trans. Amer. Math. Soc. 185 (1973), 331-344. MR0338833

[2] Earl Berkson and Ahmed Sourour, The Hermitian operators on some Banach spaces, Studia Math. 52 (1974), 33-41. MR0355668 (50 \#8142)

[3] Frank F. Bonsall and John Duncan, Complete normed algebras, Ergebnisse der Mathematik und ihrer Grenzgebiete, Band 80, Springer-Verlag, New York, 1973. MR.0423029 (54 \#11013)

[4] K. de Leeuw, Banach spaces of Lipschitz functions, Studia Math. 21 (1961/1962), 55-66. MR0140927 (25 \#4341)

[5] Klaus-Jochen Engel and Rainer Nagel, A short course on operator semigroups, Universitext, Springer, New York, 2006. MR2229872(2007e:47001)

[6] Richard J. Fleming and James E. Jamison, Isometries on Banach spaces: function spaces, Chapman \& Hall/CRC Monographs and Surveys in Pure and Applied Mathematics, vol. 129, Chapman \& Hall/CRC, Boca Raton, FL, 2003. MR1957004 (2004j:46030)

[7] Irving Glicksberg, Stone-Čech compactifications of products, Trans. Amer. Math. Soc. 90 (1959), 369-382. MR0105667 (21 \#4405)

[8] Kenneth Hoffman, Banach spaces of analytic functions, Prentice-Hall Series in Modern Analysis, Prentice-Hall Inc., Englewood Cliffs, N.J., 1962. MR0133008 (24 \#A2844)

[9] William Hornor and James E. Jamison, Isometries of some Banach spaces of analytic functions, Integral Equations Operator Theory 41 (2001), no. 4, 410-425, DOI 10.1007/BF01202102. MR1857800 (2002h:46035)

[10] James E. Jamison, Bicircular projections on some Banach spaces, Linear Algebra Appl. 420 (2007), no. 1, 29-33, DOI 10.1016/j.laa.2006.05.009. MR2277626 (2007m:47038)

[11] Krzysztof Jarosz, Isometries in semisimple, commutative Banach algebras, Proc. Amer. Math. Soc. 94 (1985), no. 1, 65-71, DOI 10.2307/2044953. MR781058 (86d:46044)

[12] E. Mayer-Wolf, Isometries between Banach spaces of Lipschitz functions, Israel J. Math. 38 (1981), no. 1-2, 58-74, DOI 10.1007/BF02761849. MR599476 (82e:46036)

[13] N. V. Rao and A. K. Roy, Linear isometries of some function spaces, Pacific J. Math. 38 (1971), 177-192. MR0308763 (46 \#7877)

[14] Donald R. Sherbert, Banach algebras of Lipschitz functions, Pacific J. Math. 13 (1963), 1387-1399. MR0156214 (27 \#6143) 
[15] Donald R. Sherbert, The structure of ideals and point derivations in Banach algebras of Lipschitz functions, Trans. Amer. Math. Soc. 111 (1964), 240-272. MR0161177 (28 \#4385)

[16] I. M. Singer and J. Wermer, Derivations on commutative normed algebras, Math. Ann. 129 (1955), 260-264. MR0070061 (16,1125c)

[17] L. L. Stachó and B. Zalar, Bicircular projections on some matrix and operator spaces, Linear Algebra Appl. 384 (2004), 9-20, DOI 10.1016/j.laa.2003.11.014. MR2055340 (2005a:46146)

[18] Marc P. Thomas, The image of a derivation is contained in the radical, Ann. of Math. (2) 128 (1988), no. 3, 435-460, DOI 10.2307/1971432. MR970607 (90d:46075)

Department of Mathematical Sciences, The University of Memphis, Memphis, TenNESSEE 38152

E-mail address: mbotelho@memphis.edu

Department of Mathematical Sciences, The University of Memphis, Memphis, TenNESSEE 38152

E-mail address: jjamison@memphis.edu

Departamento de Álgebra y Análisis Matemático, Universidad de Almería, 04120 Almería, Spain

E-mail address: ajimenez@ual.es

Campus Universitario de Puerto Real, Facultad de Ciencias, Nuestra Universidad, 11510 Puerto Real, CÁdiz, Spain

E-mail address: moises.villegas@uca.es 